ficial results are generally more rapid than in the case of many other diseases.

All sorts of objections have been, and still are, urged against the use of cold in scarlet fever. Danger is said to arise from "driving in the rash," from internal congestion, from the rapid loss of body temperature and consequent depression, and it is also said that the risk of renal mischief is thereby increased. If it were proved that the occurrence of nephritis is more frequent after treatment by cold it would be a very valid objection to the practice of it, and the real truth can only be learned by an examination of a large number of cases. From my own experience $I$ am inclined to disbelieve that any harm results in this way. The fear of the occurrence of nephritis from this cause originates no doubt in the generally accepted opinion that the affection of the kidneys so commonly occurring after scarlet fever is due to "draughts," or "catching cold," or to leaving bed too soon. I think this opinion is not founded upon sufficiently good grounds, and that everyone in the habit of seeing cases of scarlet fever must of ten have seen nephritis beginning before as well as after the patient has left his bed, and as often in cases kept in warm stuffy rooms as in those in which fresh cool air has been freely admitted to the sick chamber. "Catching cold" is made to do duty as a cause of so many conditions for which we can find no better explanation, that it is adopted at once and without hesitation in order to account for any otherwise inexplicable phenomena. There appeare, at any rate, to be no good ground for assuming that cold bathing increases the chance of an attack of nephritis, and in the two cases here reported the urine did not become albuminous after repeated bathing. Objections on the ground of the trouble and increased expense in nursing are scarcely worth consideration if it is true, as I believe it is, that this mode of treatment is better than any other. The difficulty is perhaps less in treating scarlet fever than in dealing with other cases because the patients are usually young and easily lifted in in and out of the bath, but when from the weight of the patient or the weakness of the attendants it is impossible to use the bath, the patient may easily be packed in wet sheets, with or without pieces of ice placed here and there, or india-rubber bags or $\operatorname{larg} \theta$ bottles filled with ice may be placed round the patient. The bath gives better results than any other plan when it can be thoroughly carried out, and the most satisfactory way is to begin with the bath at $98^{\circ}$ or $100^{\circ}$, and cool down gradually to about $70^{\circ}$. It is of course better that the tem. perature of the patient and of the water should be frequently taken, but the hand is generally a good enough guide as to the water, and the appearance of the patient always indicates the improvement in his condition. This is well illustrated in the case of S. F- (aged eight), near the end of the fifth day. The little patient was then drowsy and delirious, the temperature being $104^{\circ}$, and threequarters of an hour in a bath, beginning at $90^{\circ}$ and cooled down to $68^{\circ}$, resulted in the cessation of delirium and drowsiness, and a reduction of the temperature (in axilla) to $95^{\circ}$. In the case of M. A. S- (aged four), a similar bath for one hour reduced the temperature from $1053^{\circ}$ to $956^{\circ}$. There was no dangerous depression or bad symptom whatever from this low temperature. In scarlet fever, as in other allied disease, the cardiac impulse and the character of the heart sounds are safer guides as to the condition of the heart and circulation than is the pulse at the wrist, the latter being often very deceptive.

In the case of S. F- it will be noticed that the temperature rose again and again after removal from the batb, the rise beginning sometimes immediately, at others after an hour or less. This rapid rise is usual in severe cases, and the benefit derived seems always to be in proportion to the length of time during which the high temperature is warded off. It is worth consideration whether it will not ultimately turn out to be a better plan to keep the patient in the bath for many hours, even for a day or longer if necessary, cooling the water very gradually down, and maintaining it at whatever degree is found to keep the patient's temperature nearest to a healthy point. In the treatment of diseases or injuries of the skin, no evil, but, on the contrary, great good results from much more prolonged baths; and it seems far more reasonable to prolong the period of immersion than to take the patient out and allow the temperature to rise, to be again and again reduced in the same way. I am well aware that unpractised writers like myself of ten seem to be regardless of the time of their professional brethren, but, $I$ trust that the above remarks will not be considered too long nor be found altogether useless. The notes of the cases are, I think, sufficiently detailed and clear to need no furtber explanation. The first case was one of more than usual severity; and the second, though not quite so serious, was yat by no means a mild case, as a temperature of over $105^{\circ}$ and a pulse of extreme rapidity indicate.

I should add that all the baths were begun at a comfortably warm temperature-between $90^{\circ}$ and $100^{\circ}$,-and cooled down slowly to about $70^{\circ}$, or sometimes a few degrees below that.

(To be concluded.)

\section{A NEW MODE OF TREATING CERTAIN TUMOURS OF THE LYMPHATIC GLANDS.}

\section{Br S. MESSENGER BRADLEY, F.R.C.S.}

AT the recent Edinburgh meeting of the British Medical Association a paper of mine was read in which $I$ advocated the subcutaneous injection of iodine in certain cases of lymphatic tumours, and as I have since then employed this method in several fresh cases $I$ have thought that a brief a:scount of the mode of procedure might prove interesting. It must be premised that I do not speak of lymphatic tumours generally, but of certain kinds only; thus I do not refer to syphilitic or carcinomatous affections, or to the infectious or soft form of lymphomata, but confine my attention to three groups. First, true hypertrophies of the lymphatic glands, with or without a strumous diathesis second, strumous hypertrophies-i.e., cases of cellular hyperplasia plus caseous deposit; and, third, hard non-infectious lymphomata, which present many points of resemblance to the first groups, and, indeed, are often only distinguishable in being multiple.

Now, there is perhaps nothing more common than to paint iodine over all the above-mentioned tumours unless it be the disappointment which results. This, at least, is my experience, the result apparently being the same whether the iodine is painted indiscriminately over the whole gland or whether it is applied, according to Farneaux Jordan's advice, over the contiguous lymphatics rather than over the gland itself; and yet all that seems to stand between this treatment and success is the thin skin which intervenes between the gland and the pigment.

The first case in which I injected iodine into a tumour did not appear very promising, though it proved perfectly successful. It was an encapsulated tumour, about the size of a large walnut, situated beneath the lower jaw, which I should have removed with a scalpel, had I not once had some unpleasant hæmorrhage in a vrecisely similar case ; and as the patient in the present instance lived at some distance, I resolved to try to procure absorption before resorting to extirpation. The tumour almost disappeared with the first injection, and after one more it could not be at all detected. I was pleased with the result, because it appeared to me to be so desirable to adopt such a plan at one's consulting-rooms, and in the out-patient room of the hospital, instead of using the knife, which is always more or less terrible to the patient, and which is sometimes, in the most careful hands, followed by unfortunate results. Since the case I mention I have been in almost the daily habit of employing iodine in this manner, and I think I may venture to affirm that, by properly selecting cases, a successful result may be assured, while there is no doubt that an indiscriminate nse of the remedy will be productive of disappointment. The best cases are those where a single cervical gland is hypertrophied in an otherwise healthy (adult) subject. Five or six injections of the simple tincture of iodine (five to ten minims at a time, according to the size of the tumour), at intervals of about four days, generally effect a cure. The earlier stages of strumous bypertrophies are also very successfully treated by this method, 
as are the small hard multiple lymphomata; but in the later stages of strumous disease of the cervical glands, where the tumour is broken down into a mass of caseous matter, and the neighbouring skin is blue and undermined, no good results follow from the injection of iodine; and, indeed, these cases are best treated by a careful excision of the disorganised and degenerated glands. I have also recently employed iodine injections in a large and hard fibroid bronchocele, which had been treated unsuccessfully by the internal administration of the drug. The tumour was not only inconvenient from its size, but had almost destroyed the voice, and so pressed on the trachea as to deflect it to the right side of the neck. The case is still under treatment, but the first two injections of ten minims of iodine were followed by the diminution of an inch in the girth of the neck. By parity of reasoning we may expect this method to prove serviceable in uterine myomata and allied growths, but it is to its value as a remedial agent in cases of lymphatic enlargement of the cervical glands that I especially wish to call attention, and I may briefly summarise my results on this head by a tabular statement:-

1. Cases of cervical tumours to be treated by injection of iodine.

$a$. True hypertrophies of the lym phatic glands without strumous admixture.

b. Strumous hypertrophies before breaking down.

c. Hard lymphomata.

d. Encapsulated cervical tumours, as a tentative operation.

2. Cases of cervical tumours to be treated by incision.

a. Strumous glands which have broken down into pus, with or without previous treatment by injection.

3. Cases of cervical tumours to be treated by excision.

a. Strumous glands infiltrated with caseous matter, which may be rocked to and fro upon a base of degenerated cellular tissue, with a margin of blue undermined integument.

b. Encapsulated tumours which have resisted the Manchester. treatment by injection.

\section{O X A L UR I A.}

B т JAMES REOCH, M.A., M. B. (Concluded from page 310.)

These earthy salts are of three chief kinds-carbonates, phosphates, and oxalates. The carbonates may be dismissed, because, though common in decomposing urine, they do not appear in ordinary urine when voided. The phosphates also, though always present, never seem to be in sufficient quantity to exhibit insolubility in alcohol. If the average quantity of lime be $3 \frac{1}{2}$ grs. in $52 \mathrm{oz}$. of urine, it will be found on trial that two or three times this amount, in the state of acid phosphate, will give only a faint turbidity when alcohol is added to its watery solution. We are therefore thrown back on the oxalates as the chief source, if not of the white precipitate which immediately appears when alcohol is added to urine, at least of the permanent turbidity which results on shaking the mixture. But this is never a good way of obtaining oxalates; for, being thrown down quickly, they are in the form of granules, and never assume the envelope shape, however long they are afterwards allowed to stand. The alcohol ought always to be added gently to the top of the urine, and the whole allowed to stand for twenty-four hours. If the urine possesses oxalates in abundance they will of course be thrown down without the alcohol, and if it has none at all the alcohol cannot make any appear; but the vast majority of urines are placed between these extremes, and therefore give few or no oxalates unless alcohol be added. It is easily calculated that an ordinary microscopical preparation covers 10,000 times the surface which is taken in at one field of view under a power of 300 diameters; but, since it is rapidly passed over, suppose it to be only 100 times the size, and the effect of alcohol in precipitating oxalates will be appreciated when it is said

that, of 41 urines examined, there was an average of several oxalates in each field of view in nearly every one to which alcohol was added, whereas without it there was often scarcely an oxalate to be found in a whole preparation. To be more particular : from $10 \mathrm{cc}$. to $15 \mathrm{cc}$. of 36 specimens of urine from the same person, taken indiscriminately at three or four different periods of the day, were put into some test-tubes; half of them had 3 cc. rectified spirit added to them gently; the next day the excess of urine was poured off, and the few drops at the bottom examined microscopically. Where oxalates were seen, their average size and number were noted and reduced to the uniform standard of $\frac{1}{8000}$ in. in the side. The following table expresses the comparative results obtained :-

No. of urines examined No spirit. Spirit. No. of times oxalates were found in twenty- $\} \quad 30 \quad \ldots \quad 35$ $\left.\begin{array}{llllllll}\text { four hours } & \ldots & \ldots & \ldots & \ldots & \ldots & \ldots & \ldots\end{array}\right\}$

No. of times one average oxalate of $\frac{1}{3000}$ in. ? was found in each field of view ... $\ldots$...

each field of view ...

$16 \quad \ldots \quad 33$

$1 \quad \ldots \quad 15$

This table indicates the effect of adding alcohol to urine, and it may be here observed that this explains a well-known fact which has not been hitherto fully accounted for. Every surgeon knows the evil effect of alcohol in cases of stricture, but the cause is not so clear. Congestion of the mucous membrane is an unsatisfactory because merely theoretical explanation, but the greater tendency to precipitation of oxalates explains matters perfectly. If a crystal of uric acid or triple phosphate be gently pressed under the microscope it will be reduced to powder, but an equal or even greater pressure has no effect on oxalates; they are therefore comparatively hard, and as they present six solid sharp angles, each of which is finer than the sharpest needle, they must inevitably tend to irritate the already diseased blad. der and urethra. But, however this may be, the fact remains that addition of alcohol to urine in a gentle manner causes increased precipitation of oxalate of lime, and this may serve to throw some doubt on the alleged fact that carbonated drinks increase the amount of oxalates. It is natural to suppose, seeing the almost universal prevalence of oxalic acid in the vegetable kingdom, and its simple relation to $\mathrm{CO}_{2}$, that it could easily be formed from $\mathrm{CO}_{2}$; but this is not the case, and the facts above related show that it is to the alcohol, and not to the $\mathrm{CO}_{2}$, we must look for an explanation of the oxalates; not that the alcohol forms the oxalates in any way, but merely that it throws them down more completely.

The alcoholic method of analysis may be applied to determine the amount of oxalates present in the urine. Thirty specimens of different urines (10 cc. to $15 \mathrm{cc}$. of each) gave an average of twelve oxalates of $\frac{1}{200}$ in. in each field of view. All the oxalates were in abuut two to five drops at the bottom; there might therefore be from 20,000 to 100,000 of such crystals in 15 ce., or about $2,000,000$ to $10,000,000$ in $52 \mathrm{oz}$. of urine. The whole quantity probably does not nearly amount to $1 \mathrm{gr}$. per diem; but it must be confessed that the absolute measurements obtained by this method involve some data open to question, yet comparative results of great value may be obtained. Although, therefore, not able to give exactly the quantity of oxalates passed per diem, the following results give the comparative quantity at different periods. Ten specimens of "urina sanguinis" gave an average of $8 \frac{1}{2}$ oxalates of $\frac{1}{5000}$ in. in each field of view, whereas ten specimens of urine after breakfast on the same days gave an average of 22 similar crystals. They appear therefore to be increased by food, but in a very irregular manner, as the following table will show.

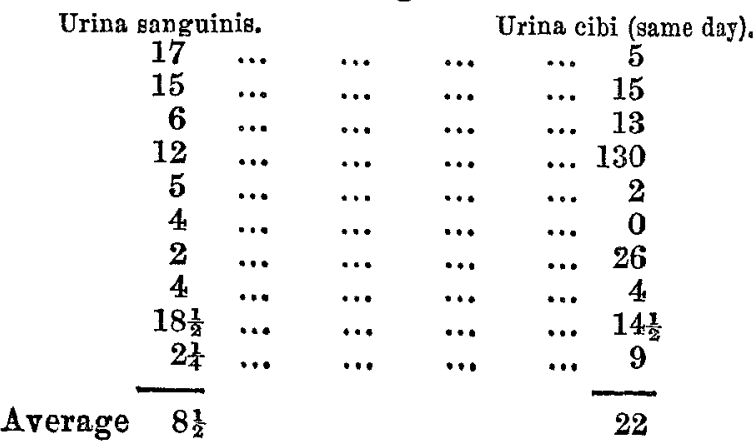

